\title{
Non Adherence to Diabetic Medication Among Diabetic Patients, a Case Study of Dormaa Hospital Ghana
}

\author{
Awuni Prosper Mandela Amaltinga \\ Department of Nursing, Nyaho Medical Center, Accra, Ghana West Africa \\ Email address: \\ mandelaprosper@yahoo.com,mawuni47@gmail.com, basyonde@yahoo.com
}

\section{To cite this article:}

Awuni Prosper Mandela Amaltinga. Non Adherence to Diabetic Medication Among Diabetic Patients, a Case Study of Dormaa Hospital Ghana. Science Journal of Public Health. Vol. 5, No. 2, 2017, pp. 88-97. doi: 10.11648/j.sjph.20170502.15

Received: July 6, 2016; Accepted: August 1, 2016; Published: February 15, 2017

\begin{abstract}
Today, diabetes Mellitus is one of the chronic diseases prevalence in Ghana. This study assessed the factors that contribute to non-adherence to medication among diabetic patients undergoing therapy in Dormaa Hospital. The study used convenience and purposive sampling technique to sample 130 respondents comprising 123 diabetic patients and 7 health professionals from the Dormaa Presby Hospital. A standardized questionnaire was used to collect the data. Data was coded and analyzed with Stata 12.0. Most participants (57\%) had a family history of diabetes and were at age 51 and above that most people were diagnosed with the disease. The level of diabetics' adherence to diabetic treatment regimen was satisfactory and higher (68.5\%). The majority of diabetics (69.23\%) visited health facilities on monthly basis for review which involves testing of blood glucose level as well. Though most of diabetics surveyed were illiterate, they were, however, knowledgeable on the importance of treatment regimen as they disclosed that treatment regimen sought to prevent progression of the disease stage to associated complications, co-morbidities, and early death. Polyuria, polydipsia, and sweating were some of the commonest sign and symptoms mentioned by the patients. The majority of the patients (57\%) had no idea of the normal range of fastening blood sugar level. Finances $(25.6 \%)$, forgetfulness $(45.5 \%)$, busy schedules $(18.2 \%)$ and poor family support $(2.5 \%)$ were some factors that affect better treatment adherence. Most diabetics called for a government subsidy to the drugs, continuous health education on the diseases and family support. The study recommends a much more focused preventive healthcare services to identify risk factors and early detection. Research on the burden of chronic diseases on the health facilities should be considered in the future.
\end{abstract}

Keywords: Nonadherence, Diabetes, Random Blood Sugar (RBS), Poliuria, Polydepsia, Co-morbidities, Komfo Anokye, Korle Bu, Hypogylcaemia

\section{Introduction}

\subsection{Background to the Study}

Non-adherence to medical treatment remains a challenge for the medical professionals and social scientists. Their efforts to explain and improve patient adherence often appear to be ineffective. Although successful adherence interventions do exist, $50 \%$ of interventions seem to fail according to Arifulla et al. (2014). Anti-diabetic medications are integral for glycaemic control in diabetes, therefore non-adherence to drugs can alter blood glucose level resulting in complications (Arifulla et al., 2014). Consistent adherence among patients with chronic conditions is disappointingly low, dropping most dramatically after the first six months of therapy (Varney et al., 2008).
Simpson et al. (2009) classified the interventions in four mainstream, global, theories: behavioural, educational, effective, or combinations of these. For example, when financial incentives are being used to improve adherence, the underlying theoretical perspective is behavioural because incentives are considered to act as positive re-enforcers. Another class of intervention focuses on persuasive communication to improve adherence. Poor adherence has been shown by McDonald et al. (2011), to decrease the effects of prescribed medications or other treatments and to increase the likelihood of poor outcomes.

\subsection{Statement of the Research Problem}

According to Wabe (2011), Diabetes is a chronic disease for which control of the condition demands patient self-management. Self-management behaviours include 
monitoring blood glucose levels, taking medication, maintaining a healthy diet and regularly exercising. For most patients, it is important to conduct daily foot exams. Diabetes peer support groups are a valuable source of diabetes information and link patients together to provide mutual support (Horne, 2007).

For this reason, the Ghana Diabetes Programme was initiated in 1995 with the goal of increasing and facilitating access to diabetes care and to ensure the sustainability of the programme. A Diabetes Centre was run once a week at Korle $\mathrm{Bu}$ Teaching Hospital in Accra. In 1990 a second diabetes Centre was set up in Kumasi at Komfo Anokye Teaching Hospital to cater for the Northern part of the country (Buabeng et al., 2008).

Despite all these interventions, Prescribers uniformly underestimate the problems of non-adherence in their patients. If a health care professional is unable to detect non-adherence, it is impossible for him or her to correct the problem. Hence it becomes imperative to measure and evaluate patient adherence reliably (Roth et al., 2007). There are relatively high levels of non-adherence in all areas of self-management behaviors. This non-adherence is perhaps due to the fact that self-management behaviors usually require changes in the patient's daily life according to Buabeng (2008).

According to the WHO (2011) data, Diabetes Mellitus deaths in Ghana reached 2,752 or $1.47 \%$ of total deaths. The age-adjusted death rate is 23.47 per 100,000 of the population thus ranks Ghana 111th in the world. It further points that, the national prevalence of diabetes is $3.35 \%$. The number of males living with diabetes in Ghana is estimated at 223,000 and the females estimated at 216,000, whilst that of an urban setting is estimated at 233,000 .

A recent analysis of admissions in the district hospitals of the country in which Dorman Presby hospital is inclusive reveals that diabetes accounts for $6.8 \%$ of all admissions. The data also indicates the number of people living with diabetes in the rural Ghanaian setting like Dormaa Ahenkro is estimated at 206,000 (MOH, 2013).

Non-adherence to diabetic treatment among patients in rural communities in Ghana where there is a lack of access to health service, ignorance and poverty subsequently causes cardiovascular diseases like hypertension and stroke, chronic renal disease, retinopathy and sometimes spontaneous sudden death among such patients (Buabeng et al., 2008).

Treating chronic disease in Ghana is expensive, without health insurance. Managing a condition such as diabetes can cost more than the average individual earns. The economic impact of chronic diseases on individuals has a knock-on impact on their family livelihood and relations, as well as their long-term treatment choices (De-Graft, 2007). A rural-urban study of diabetes experienced by Ghanaians showed that many poor rural men and women with diabetes have difficulty in adhering to treatment because they relied on financial support from their immediate and distant family members (De-Graft 2007).

In view of the consequences of non-adherence to anti-diabetic medicines, this study seeks to evaluate the magnitude of non-adherence of diabetic patients in the Dormaa Presby hospital, and its related causes. These can be used to increase awareness among medical officers, prescribers, other health professionals and managers of healthcare to develop strategies to minimize non-adherence to anti-diabetic medication.

\subsection{Justification of the Study}

Drugs or medications constitute a vital component in the management of diabetes, but it is a fact that a lot of diabetic patients find it difficult to adhere to their drugs regimen.

The researcher observed that higher percentage of diabetes patients are not adhering to their treatment few months after discharge from the hospital. They would come back in a worsening condition and have to be hospitalized for some days progressing to several weeks. Is it because of side effects, drug overdose dose or cost related? These and much more are what prompt a research into this subject.

The findings will help the government, Non-governmental organizations, Health Training Institutions and the society at large strategize appropriately to enable them to work efficiently to deal with the problem of non-adherence to medication among Diabetic patients.

\subsection{Research Questions}

1. What is the level of adherence of diabetic patients to the treatment regimen in Dormaa Presby Hospital?

2. What is the level knowledge of diabetic patient on the disease management?

3. What probable factors are associated with non-adherence to diabetic treatment regimen?

4. How can adherence to medications of diabetic patients be improved?

\subsection{Research Objectives}

Based on the afore-mentioned factors associated with non-adherence to medications, the study focused on the following objectives:

\subsubsection{General Objective}

To assess the factors that contributes to non-adherence to medication among diabetic patients undergoing therapy in Dormaa Hospital.

\subsubsection{Specific Objectives}

1. To assess the non-adherence rate of diabetic patients in the Dormaa Presby Hospital.

2. To assess the knowledge level of diabetic patients in relation to the disease management.

3. To identify factors responsible for non-adherence to anti-diabetic therapy

4. To identify effective adherence interventions for anti-diabetic therapy.

\subsection{Significance of the Study}

This study is geared towards exploring factors associated with non-adherence to medications among diabetic patients with the aim of identifying lapses in the drug regimen in 
order to aid stakeholders, health professionals, and health facilities provide effective and efficient care to clients and ensure their adherence to the treatment regimen.

Consequently, information generated from this study would help people in their scholarly research theory or practice as they will get to know the behavior of patients towards treatment regimen in Dormaa Presby Hospital.

\subsection{Scope of the Study}

The study is limited to diabetic patients accessing health from the Dormaa Presby Hospital as its inclusive criteria with the aim of assessing factors associated with non-adherence to medications among diabetic patients and to make recommendations to the relevant bodies for improvement in the adherence rate of medication among patients diabetes.

\subsection{Organisation of the Chapters}

The entire research was divided into five chapters. Chapter one covered the introduction of the research. Chapter two covered the literature review on medication non-adherence and specifically on diabetes patients. It also described the empirical studies that have previously been carried out. Chapter three described the methodology; it discussed the study area, study type and design, the study population, the sampling size and sampling techniques, data collection tools and techniques and data processing and analysis. The ethical issues and limitation of study were also addressed in this chapter. Chapter four covered the presentation of results and discussion. Conclusions and recommendations made up the chapter five.

\section{Literature Review}

\subsection{Introduction}

Neumann (2010) asserts that literature review is based on the assumption that knowledge accumulates and that we learn from and build on what others have done. This chapter provides the theoretical framework and the empirical evidence of related work for the study.

\subsection{Prevalence of Not-adherence to Diabetic Medication Globally}

Worldwide, adherence to medication for diabetes varies between 36 and 93\% according to Wabe (2011). Adherence to prescribed medication is crucial to reach metabolic control as non-adherence with blood glucose lowering or lipid lowering drug is associated with higher HbAIc and cholesterol, levels respectively (Ramphal et al., 2008). A study conducted in Netherland on refill adherence and Polypharmacy among patients with type- 2 diabetes in general practice showed that mean adherence with oral glucose-lowering drugs is between 61 and $85 \%$. According to the study, it has become apparent, an increase in the number of co-medications tends to decrease the adherence of patient with type-2 diabetes to their treatment regiments (Horne, 2008).

According to Centre for disease control and prevention, (CDC, 2011) 64.7\% all newly diagnosed with diabetes were an adult with ages 50 and above.

Poor adherence to treatment of chronic disease is a worldwide problem of striking magnitude. Recently, the world health organization stated that only $50 \%$ of a patient diagnosed with chronic illness were fully compliant with their treatment regimen, in developing country the rate are even lower. It is undeniable that many patient experiences difficulty in following treatment recommendations (Wabe, 2011).

\subsection{Prevalence of Non-adherence to Diabetic Medications in Africa}

A study on the adherence to anti- diabetic drug therapy and self-management practice among type- 2 diabetics conducted in southwestern Nigeria showed that about two third of patients representing 33\% were not adherent according to Kolawole et al. (2008). Furthermore, studies carried out in developed country like Uganda, revealed a gap in knowledge about the prevalence and factors that may be associated with non-adherence to diabetic treatment in Uganda (Kalyango et al., 2008).

Adewale et al. (2008) stated that 30\% Patients diagnosed with type 2 diabetes mellitus in Extension II Clinic in Botswana have difficulty in adhering to the lifestyle modifications recommended by healthcare practitioners. Poor adherence to lifestyle recommendations leads to poor control of the condition and consequently to complications.

\subsection{Non-adherence Rates of Medication Among Diabetic Patients}

The World Health Organization defines adherence to long-term therapy as the extent to which a person's behavior taking a medicine, following a diet, and or executing lifestyle changes-corresponds with agreed recommendations from a healthcare provider (WHO, 2008).

Adherence has been also defined as the "active, voluntary, and collaborative involvement of the patient in a mutually acceptable course of behavior to produce a therapeutic result" (Meichenbaum, 2013). A recently published article by Odegard et al.(2007) indicated that adherence rates to diabetes medication varied from $31 \%$ to $87 \%$ in retrospective studies and 53 to $98 \%$ in prospective studies. Approximately $50 \%$ of diabetes patients are non- adherent when it comes to taking diabetes-related medications according to study by Heisler (2008).

Adherence is measured in a variety of ways including pill counts, self-reports or patient diaries, physician reports, reports by others (such as the patient's spouse), electronic measures (e.g., metered dose inhalers or electronic recordings of dispensed eye drop); blood or urine assays, medical records and pharmacy records. Direct observation of a patient taking medication is another, albeit more 
energy-intensive, method for assessing adherence (Volmink et al., 2010).

\subsection{Knowledge of Diabetic Patients in Relation to Treatment Regimen}

Diabetes education is widely accepted as integral to diabetes therapy within the diabetes community. Priority is given by WHO for the development of diabetes education program in many areas to give patients a better knowledge of their disease. In order to prevent premature morbidity and mortality associated with diabetes awareness and educational programs should be promoted (Adil et al., 2010).

The education of diabetic patients, proposed as an essential therapeutic tool since the early 1920 s, has generated great enthusiasm over the last decade, with increasing concern for greater effectiveness by improved motivation of both patients and doctors. Structured education depends on the precise definition of agreed, short-term objectives, whose attainment shall be verified. Educational objectives may be set at different levels: knowledge of the disease, skills required for treatment and capacity to integrate therapy in everyday life. Patients' motivation to learn and adhere to treatment is also greatly influenced by individual factors, both psychological and environmental, that need to be taken into account (Hansotia, 2013).

A study conducted in Pakistan at Foundation Clinic, Shifa College of Medicine, Islamabad, in April 2004 showed low level of awareness among the participants. Only $6(15 \%)$ of the study participants could correctly state their fasting blood sugar level. Knowledge about HbA1c was poor. Only 4 (10\%) ever had their HbA1c level checked and only one patient could tell the normal reference level of HbA1c. Knowledge about the complications of diabetes such as heart attack, stroke, eye and foot complications was less than 50\% (Adil et al., 2010).

\subsection{Factors Responsible for Not-adherence to Diabetic Therapy}

Demographic, psychological, and social factors, as well as health care provider, medical system, and disease- and treatment-related factors improve patient adherence

\subsubsection{Demographic Factors}

Demographic factors such as ethnic minority, low socioeconomic status, and low levels of education have been associated with lower regimen adherence and greater diabetes-related morbidity, for example, lower rates of Self-monitoring of blood glucose (SMBG) have been observed among minority African-American and Mexican-American patients. A recent study using a large national sample of patients with type 2 diabetes found that $24 \%$ of insulin-treated patients, $65 \%$ of those on oral medications, and $80 \%$ of those treated by diet and exercise alone either never performed SMBG or did so less than once per month. Daily SMBG (at least one blood glucose check per day) was reported by only $39 \%$ of patients treated with insulin and just $5 \%$ of those treated with either oral medications or diet and exercise (Delamater, 2008).

In the face of financial constraints, diabetic patients may forgo prescribed medications, causing negative health effects. Financial variables especially the direct and indirect costs associated with a prescribed regimen and restricted access to therapy have been found by several studies to influence patients' commitment to medication adherence in developing countries (Boabeng et al., 2012). A study by Madden et al. (2013) revealed that older adults skip doses, reduce doses, or do not get prescriptions filled because they cannot afford to pay for medication

Anderson et al. (2008) also claimed demographic factors such as ethnic minority, low socioeconomic status, and low levels of education have been associated with lower regimen adherence and greater diabetes-related morbidity. Socioeconomic factors play a vital role in adherence, as patients who are poor or live on a fixed income may be non-adherent because of their inability to afford the cost of prescribed medications. The research also showed that patients who have limited access to transportation, live far away from clinics may not likely adhere to treatment regimens

\subsubsection{Psychological Factors}

Psychological factors are also linked with regimen adherence. Appropriate health beliefs, such as perceived seriousness of diabetes, vulnerability to complications, and the efficacy of treatment, can predict better adherence. Patients adhere well when the treatment regimen makes sense to them, when it seems effective, when they believe the benefits exceed the cost, when they feel they have the ability to succeed at the regimen, and when their environment supports regimen-related behaviors (Delamater, 2008).

Higher levels of stress and maladaptive coping have been associated with more adherence problems. Psychological problems such as anxiety, depression, and eating disorders have also been linked with worse diabetes management in both youths and adults with diabetes. A Diabetes Attitudes Wishes and Needs (DAWN) study in the United States of America showed that a significant number of diabetic patients have poor psychological well-being and that providers reported that these psychological problems adversely affected regimen adherence. This study also showed that many health care providers do not feel confident in their ability to identify psychological problems in their patients or to provide the psychological support their patients need (Peyrot, 2010).

Appropriate health beliefs, such as perceived seriousness of diabetes, vulnerability to complications, and the efficacy of treatment, can predict better adherence according to Brownlee-Duffeck (2011). This is because Patients adhere well when the treatment regimen makes sense to them, when it seems effective, when they believe the benefits exceed the costs when they feel they have the ability to succeed at the regimen. Tjia (2008), in a study to examine the concerns of older adults with diabetes about the complexity of their medication regimen and whether they discussed 
medication-related concerns with their physician found that many older adults did not think it was appropriate to discuss their concerns about medication with their physician or doubted that chronic illness medications were necessary.

\subsubsection{Social Factors}

Family relationships play an important role in diabetes management. Studies have shown that low levels of conflict, high levels of cohesion and organization, and good communication patterns are associated with better regimen adherence. Greater levels of social support, particularly diabetes-related support from spouses and other family members are associated with better regimen adherence. Social support also serves to buffer the adverse effect of stress on diabetes management (Al-Qazaz, 2011).

Past research has indicated three main types of social support. The three types are emotional support, instrumental support, and informational support. Emotional support is the most widely studied and is defined by someone receiving love, encouragement and having an individual in whom to confide concerns. Instrumental support includes receiving material help, such as financial aid and/or household help. Information support is defined as a person receiving information about their specific needs and/ or condition (Hamilton, 2009).

\subsubsection{Healthcare Provider and Medical System Factors}

Social support provided by nurse case managers has been shown to promote adherence of diabetic patients to diet, medications, SMBG, and weight loss. Also having regular, frequent contact with patients by telephone promoted regimen adherence and achieved improvements in glycemic control, as well as in lipid and blood pressure levels. It was observed in the Diabetes Control and Complications Trial that one of the key elements to success in achieving good glycemic control was the availability of support provided to patients by the healthcare team (Anderson et al., 2008).

In addition to the ability to obtain support from health care team members, the quality of the patient-doctor relationship is a very important determinant of regimen adherence. Patients who are satisfied with their relationship with their health care providers have better adherence to diabetes regimens. In addition, patients who have a "dismissing attachment" style (discomfort trusting others ) and therefore greater self-reliance (positive view of self) toward their doctor and who rate their patient-provider communication as poor have been shown to have lower adherence rates to oral medications and SMBG. Organizational factors that promote adherence include reminder post cards and phone calls about upcoming patient appointments that begin on time (Harris, 2013).

Patient satisfaction is important because it enhances health-seeking behaviours in individuals, improves treatment compliance, and fosters the relationship between patient and health professional (Spear, 2010). The extent to which consumer opinion can influence policy makers and health care personnel is not only dependent upon collecting the right kind of data, it also requires that policy and health personnel accept the value of the consumer's point of view (Locker, 2013).

\subsubsection{Disease and Treatment-Related Factors}

Research has generally shown that lower regimen adherence can be expected when a health condition is chronic, when the course of symptoms varies or when symptoms are not apparent when a regimen is more complex, and when a treatment regimen requires lifestyle changes. Studies with diabetic patients indicate better adherence to medications than to prescribed lifestyle changes and better adherence to simpler regimens than to more complex ones (Paes, 2007).

\subsection{Effective Adherence Interventions for Anti-diabetic Treatment Regimen}

Ironically, to improve diabetic patients' compliance or adherence, health care professionals should first abandon the concept of trying to get their patients to comply or adhere better. This requires an attitude shift in recognition of patient responsibility for diabetes self-management, as well as a new type of collaborative relationship with patients. There is no question that diabetes management can be frustrating for health care providers, but it is important to be aware of how these attitudes may determine approaches to clinical practice and undermine effective diabetes management (Peyrot, 2010).

\subsubsection{Traditional Approach to Health Behavior Change}

In the traditional approach to health behavior change, the health care provider is seen as the expert who knows what is best for the patient; advice-giving is the technique used for the delivery of knowledge to the patient. This assumes that patients should change their behavior, want to change and that their health and their prescribed regimen are major priorities for them. However, giving advice may not be the most skillful approach to health behavior change because telling patients what to do undermine their sense of autonomy, generates resistance, may not consider what is important to patients, and does not work in the majority of cases (Larme \& Pugh, 2011).

\subsubsection{Assessment of Diabetes Management Problems}

Effective behavioral interventions first require an understanding of why, how, and when patients do not engage in optimal diabetes self-management behaviors. Assessment of the reasons for lack of optimal self-care is important before embarking on specific behavioral interventions that may fail if specific regimen barriers are not understood and dealt with as part of the intervention. Disease-related knowledge and skills may be lacking, or patients may have inappropriate health beliefs and attitudes (Claxton, 2009).

Specific environmental barriers may adversely affect patients' ability to perform appropriate self-care. Patients may be socially isolated or have conflicted family relationships that undermine diabetes management. There may be specific psychological or psychiatric disorders, such as depression, anxiety, or eating disorders that impair effective diabetes management (Anderson et al., 2008).

\subsubsection{Effective Behavioral Interventions}

Health care providers must understand behavior change as part of an interpersonal process. Although patients are 
responsible for their own decisions and self-care behaviors, patient outcomes are also affected by health care provider behaviors. To be most effective at health behavior change, health care providers should have a patient-centered approach, cultivate a collaborative relationship, communicate clearly, and provide directives (advice) when patients are ready to hear and learn more about the new recommendations (Rollnick et al., 2013).

According to the study by Haynes (2012), several specific strategies are able to help patients with the changing behavior First is the establishment of rapport, conveying genuine interest in patients. An agenda should be set in terms of talking about some specific health care goals. Providers should assess the importance patients place on and the confidence they feel with respect to specific health behaviors to determine their readiness or motivation. It is important during the clinical encounter to explore the importance of regimen-related behaviors and build patient confidence. Assuming that patients do want to hear what providers want to tell them, exchanging information is a critical part of the behavior-change process. A rationale should be provided for the recommended treatments. Therefore, it is important to remember that simply providing information to increase knowledge will not guarantee that behavior change occurs.

During the effective behavioral consultation, providers encourage patients to express their concerns and use active listening techniques, such as open-ended questions, clarifications, reflective statements, and summary statements. Health care providers help their patients to be more active, brainstorm options and consider the advantages and disadvantages of various therapeutic approaches. Collaborating and negotiating are integral to these encounters, but patients assume control over decision making (Glasgow \& Toober, 2009).

\section{Methodology}

\subsection{Study Type and Design}

\subsubsection{Study Design}

An exploratory and descriptive study design were used to assess factors affecting adherence to diabetic treatment. The qualitative and quantitative exploratory were used because very little are known about the factors causing non-adherence to treatment among diabetic patients in Dormaa Presby hospital.

\subsubsection{Study Variables}

The study collected information on socio-demographic characteristics (age, sex, marital status, and level of education, occupation, and religion) as background variables. The variables to the study include independent, dependent and background variables. The independent variables to the study include; the level of non-adherence to diabetic medication, knowledge of patients on diabetes drug management, factors associated with non-adherence to medications whilst the ways to improve adherence to antidiabetic medications in Dormaa Presby hospital is the dependent variable in this study.

\subsection{Study Population}

\subsubsection{Inclusion Criteria}

The inclusion criteria consist of respondents, who are 18 years old or above as this age is enough for people to make an informed decision of their own. Also, respondents must have diabetes and access health care from the Dormaa Presby Hospital during the study period and given written informed consent to participate in the study.

\subsubsection{Exclusion Criteria}

Non-diabetic Patients who access health care from Dormaa Presby hospital. It also excludes diabetic patients who do not seek health care from the Dormaa Presby hospital. Patients who were very ill and those newly diagnosed with diabetes (less than one month) were also excluded from the study.

\subsection{Sampling Techniques/Methods}

\subsubsection{Sampling Frame}

The sampling frame for the study was a register diabetic patient assessing health care from the Dormaa Presby hospital.

The targeted study sample for this study was 130 patients in order to be representative and large enough to be able to draw valid conclusions, to give an adequate reflection of the study population and to cater for non-responses. By ensuring an appropriate sample design as well as large enough sample size, sample error can also be reduced. The sample size was calculated using the standard formula for estimated sample size as follows:

Where $\mathrm{n}=$ sample size, $\mathrm{N}=$ Sample frame (303) and $\alpha=$ margin of error $(0.05)$ or $95 \%$

\subsubsection{Sample Technique}

The sample methods employed in this study was the non-probability sampling method. For the non-probability sampling, the researchers used convenience and purposive sampling.

The convenience sampling technique was used in selecting the hospital. This sampling method was used because the hospital has an available register for diabetic patients who attend the hospital for treatment, thereby enabling availability of data. The facility and its staff were also willing to take part in the study; this also made it easy for accessibility and use of subjects.

Purposive sampling was used to select the participants for the questionnaire in the Dorman Presbyterian Hospital, besides; the researchers chose the sample based on who they think were appropriate for the study. This method was used primarily because the participants (some health professionals) chosen were unique cases that were especially informative and knowledgeable with regards to factors affecting the adherence to the treatment regimen in the Dormaa Presby hospital. Also, the clients were members of a difficult-to-reach, specialized population (diabetics). 


\subsection{Data Collection Methods}

This study used two sources of data. These were primary and secondary data source. For primary data source, the study used questionnaires because questionnaires are advantageous while the sample size is large enough to make it uneconomical for reasons of time or funds to observe or interview every subject.

\subsubsection{Questionnaire}

A semi-structured questionnaire comprising of open and close-ended questions was designed for the study. The questionnaire collected information on demographic and socioeconomic characteristics, marriage, and knowledge of diabetes treatment regimen. Besides, the questionnaire included the patients' age, the level of education, work status and geographical location. In addition, the questionnaire collected information on the Psychological factors, Social factors and Healthcare provider and medical system factors affecting adherence to diabetes treatment. Lastly, the questionnaire included information on Disease- and treatment-related factors and other relevant household characteristics. The questionnaire was however designed by the researchers and not from elsewhere. Questions were administered to respondents in the local language for easy understanding.

\subsubsection{Use of Literature/Document Review}

A search was conducted on accessible bibliographic databases; HINARI, PubMed, and Google Scholar to identify relevant literature on the determinants of non-adherence to diabetic treatment. The keywords used to conduct the search "educational level" and "adherence to diabetic treatment", "Demography" and "ATDT (Adherence to Diabetic Treatment)", "Psychological factors" and "ATDT", "Social factors" and "ATDT"," Health care provider and medical system" and "ATDT" and "Disease- and treatment-related factors" and "ATDT". Additionally, a grey literature search was sourced via the Google internet search engine as well as newspaper articles online on ATDT were used.

\subsection{Data Analysis Methods}

The Statistical Package for Social Sciences (SPSS) version 20 and Microsoft Excel (2010) were used. The Microsoft Excel (2010) was used to plot the charts. Data was presented in tables, charts, and graphs for interpretation and analysis, based on frequency distributions, percentages, and descriptive analysis of the variables under study.

\subsection{Ethical Consideration}

After approval of the topic from the research Coordinator of the school permission to carry out the study was sought from the Medical Administrator of the Hospital. Consent of all participants was also sorted before including them in the study. Participation in the study was purely voluntary.

\subsection{Limitation of the Study}

The duration of time for the study was short. The sample size of 130 subjects is not large enough to help generalized the result. Also, Self-report method used to measure adherence in this study could overestimate adherence hence may introduce bias in the results obtained. Like many other subjective methods of measurement, it is associated with limitation. The concept of non-adherence is broad (dietary, exercise, medication etc.) as a result, the researcher limited the study to non-adherence to diabetes medication.

Moreover, this study tended to use questionnaires to assess the degree of adherence, but many questionnaires have been seen as problematic. As Whitfield et al. (2007) pointed out, 'poor questionnaires may limit the opportunity of patients to express their concerns about different aspects of care. Strictly formulated questionnaire might miss important aspects of the service experience. From the methodological point of view using a questionnaire to measure adherence may result in obtaining answers to the questions you have asked.

\section{Presentation of Results and Discussion}

\subsection{Socio-demographic Characteristics of Respondents}

The highest in terms of gender differences among the interviewees were females representing $60 \%$ and the two modal age groups were $18-45$ years and over 50 years $(38.5 \%)$. More than half $(55 \%)$ of the participants were married. Christianity was the dominant religion practiced by half of the people surveyed (50\%). The majority (70.1) of the respondents were predominantly unemployed. Nearly half (43.85\%) of the people had not received any form of education. Most of the participants $(57 \%)$ had a family history of diabetes. It was at age 51 and over that, most people were diagnosed with the disease. Indeed, DM like any other chronic diseases has sudden onset and acute stages, usually have a gradual onset and even become more prevalent with old age though it can occur at all stages of people lifespan including children (adolescent diabetes) and young; hence it is therefore not surprising that most respondents got to know of their diabetes status at late age (51 years and above). This supported one done by the Centre for disease control and prevention, (2011) who found $64.7 \%$ all newly diagnosed with diabetes were an adult with ages 50 and above.

\subsection{Level of Non-adherent to Diabetic Treatment Regimen}

Findings of the study showed higher (68.5\%) level of adherence to diabetic treatment regimen by most diabetics which are comparable to that of Wabe (2011) results that global adherence to medication for diabetes varies between 36 and $93 \%$. The present result on level of adherence $(68.5 \%)$ is about twice that of Nigeria where two third (33\%) of patients were not adherent (Kolawole et al., 2007) and contradicts Heisler (2008) findings that 50\% of diabetes patients are not- adherent when it comes to taking diabetes-related medications. Participants revealed that missing the treatment was usually unintentional because of higher awareness of the consequences that incomplete 
treatment can result in increased blood sugar level and more disabled state. Travelling was indicated as one of the situations that could prevent patients from missing their medications and therefore patients often managed to travel with their diabetes drugs. Diabetes is a chronic disease and therefore strict adherence to the treatment regimen is very significant since the medications are meant to control the level of blood sugar among diabetics being it insulin or non-insulin dependent. It was discovered that most diabetics $(88 \%)$ follow keenly the instructions of the drugs given to them by the service providers and tested their blood glucose level on a monthly basis. Almost all diabetics (97\%) visit health providers on a monthly basis for review through which they have their blood glucose level tested to find out whether the glucose level is higher than the normal range of 3.9-5.5 $\mathrm{mmo1} / 1$.

\subsection{Level of Knowledge of Diabetes Patients in Relation to Treatment Regimen}

The study findings show an absence of self-monitored blood glucose level among diabetes clients. Knowledge level among diabetics with regards to treatment regimen was low as more than half $(57 \%)$ of the patients surveyed disclosed that they had no idea of the normal blood glucose level. The result is similar to that of Adil et al., 2010) study in Pakistan where only $15 \%$ of the study participants could correctly state their fasting blood sugar level. The participants (77\%) reported knowing the signs and symptoms of diabetes mellitus and mentioned polyuria, polydipsia, blurry vision and other commonest signs and symptoms such as a headache, sweating and weakness. To most people, sugar is the preferred commodity used in correcting hypoglycaemia. Salt was also mentioned by most people as a commodity used in treating diabetes mellitus allude to the fact that health providers often advised them (diabetics) to either stop taking salt completely or reduce their intake hence the common proposition held by most clients that salt is one of diabetes drugs.

Clients envisaged the fact that diabetes drugs are importance because they aid with control of their conditions. Apart from using drugs to control diabetes, the drugs further aid in preventing progression of the disease stage to its tertiary level and its associated complications and co-morbidities which could often result in early death or conditions such as diabetes retinopathy as disclosed by participants when asked on the importance of diabetes drugs. Such findings are congruent to study of Brownlee-Duffeck (2011) that vulnerability to complications motivates diabetics to better adherence. A significant number of diabetics $(53.85 \%)$ could not indicate the type of drugs used in treating DM due to the fact that most of them had no level of education. However, most participants showed that they could identify easily single out metformin from other drugs dispensed to them by providers but have difficulty to pronouncing it.

\subsection{Factors Responsible for Non-adherence of Anti-diabetic Therapy}

The burden of chronic diseases such as diabetes mellitus is often enormous on both the patients and health providers. The survey reveals finances $(25.6 \%)$, forgetfulness $(45.5 \%)$, busy schedules $(18.2 \%)$ and poor family support $(2.5 \%)$ as major factors contributing to non-adherence of diabetes therapy confirming studies of Boabeng et al. (2012) and Madden et al. (2013) who reported financial constraints as catalyst to non-adherence to DM treatment therapy. Participants reported receiving frequent information on diabetes mellitus from physicians as indicated by $74.6 \%$ $(n=97)$ out of 130 respondents. Provider-clients communication or information sharing is very vital especially in the event of more complicated and chronic diseases such as DM. One of the factors leading to withholding of vital information relating to diseases by most clients is feeling of uneasiness in asking health professionals' questions.

This study reveals that most diabetics (57\%) felt comfortable to ask health providers the right questions pertaining to their health state or condition and were mostly $(76 \%)$ involved in decision-making process in the treatment regimen suggesting good provider-to-clients relationship confirming Spear (2010) assertion that treatment compliance often improves where there is better relationship between patient and health professional and Glasgow \& Toober, (2009). In this contemporary world, rights of the patients cannot be overlooked especially as more people are becoming enlightened with the patient charter that spells out the rights and responsibility of patients hence, involving clients in any decision-making pertaining to their health is not only in accordance to the rights of the patients but contribute to better individual health outcome especially adhering to prescribed medications and advice.

\subsection{Effective Intervention to Non-adherence to Diabetic Treatment Regimen}

Chronic diseases for which diabetes mellitus is no exception continue to be the leading cause of disability in the communities. The increasing prevalence of diabetes has a great impact on the health system, families, and the community as a whole due to the complexity of causes. Early detection and prevention of the disease DM to progress to disability stage sometimes depend on multiple factors for which intervention to increase strict adherence to diabetic treatment therapy is one of such factors.

The current study found out that health providers (prescribers) mostly believed that the best way to halt or minimize non-adherence to diabetic treatment regimen by diabetics was for them (health professionals) to decide for the patients because the patients are purported to be less knowledgeable in terms of the treatment regimen. This act of often deciding for patients with the intention that they have little inputs to make toward DM treatment regimen is not the best approach because of ever increasing patient needs. For instance, the Australian Health Ministers' Conference 2005 on National Chronic Disease Strategy call on health providers to adopt clients centres approach to achieve person-centres care. This person-centred care according to 
the paper focused on the fact that 'patient journey' comprising all of the person's experiences of health, it outcome and other factors oriented toward making a positive difference are determined by the person himself or herself (clients). Due to the impact of chronic diseases like DM and its associated stress, concerted effort to involve all players such as family members in the treatment process have to be encouraged as most health professionals $(67 \%)$ revealed that adherence to DM treatment regimen is not the sole responsibility of the patients especially when DM clients are under diets control and experiencing series of different psychological conditions.

\section{Conclusion and Recommendations}

This chapter focuses on the conclusion and recommendations of the study.

\subsection{Summary and Conclusion}

The study findings indicate an overall lack of emphasis on early detection of chronic diseases as most people get to know of their DM statuses at older ages. Preventive services seem to be missing in our current health services the trend if continue will lead to a tremendous increase in diabetes mellitus and other chronic diseases prevalence. Treatment of $\mathrm{DM}$ is somehow burdensome and therefore needs the support of all stakeholders such as patients, family, society, health providers, psychologists and many others.

\subsubsection{Level of Non-adherent to Diabetic Treatment Regimen}

Diabetics' level of adherence to diabetic treatment regimen was satisfactory and higher $(68.5 \%)$ as most of the patients knew of the effect of missing the treatment or not taking the medication at all and admitted taking the drugs often. A significant number of diabetics confirmed travelling with their DM drugs and visited health facilities on a monthly basis for review and continuation of care.

\subsubsection{Level of Knowledge of Diabetes Patients in Relation to Treatment Regimen}

The study outcomes show an absence of self-monitored blood glucose level among diabetes clients with the reason that they cannot afford the glucometer. Although most diabetics surveyed did not have any form of education, a greater number of the diabetics had knowledge on the importance of treatment regime. Most disclosed that the best way to prevent progression of the disease stage to associated complications and co-morbidities and early death was to take the DM treatment seriously. Commonest signs and symptoms of DM as mentioned by the patients were polyuria, polydipsia, blurry vision and other such as a headache, sweating and weakness.

\subsubsection{Factors Responsible for Non-adherence of Anti-diabetic Therapy}

The study discovered finances $(25.6 \%)$, forgetfulness (45.5\%), busy schedules $(18.2 \%)$ and poor family support
(2.5\%) as some factors accounting for diabetics' non-adherence to treatment therapy. Opportunities for diabetics to ask providers of the state of their health and involvement in decision making were in existence hence ruled out as factors responsible for non-adherence to anti-diabetic therapy.

\subsubsection{Effective Intervention to Non-adherence to Diabetic Treatment Regimen}

The present study showed that prescribers mostly assumed that the best way to minimalize non-adherence to diabetic treatment regimen by diabetics was to decide for them very often due to the low level of knowledge with regards to DM treatment. Some also believed that government subsidy to DM drugs could be one of the effective interventions to non-adherence to DM treatment regimen as most people find it difficult to pay for drugs especially the uninsured clients.

\subsection{Recommendations}

In view of the burden of diabetes and other chronic diseases, the study presents the following recommendations:

That early prevention and risk reduction strategy should be adopted by health providers and other health related NGOs through frequent health promotional activities to reduce the onset of the disease since the origins of many risk factors to DM are in early life.

That an effective intervention to battle the non-adherence to DM treatment regimen is to build healthy public health policy and reorienting existing health services to be to focus much on prevention and strengthen community action to understand the effect of certain lifestyle and behaviors and to support healthy lifestyle choices.

That the treatment of diabetics should not be the sole responsibilities of the patients because of the physical and psychological burden the disease brings on such victims. Efforts to have an integrated family support during the treatment process will aid in better treatment adherence and integration into the society or family.

That the reorienting of the health system and services should incorporate measures to ensure that chronic diseases such as DM services ranging from counselling to regular blood sugar level checking should be available in all basic primary health care.

Recommendations for Further Research

The study recommends that more studies should be done in other areas (outside Dormaa Municipality) to establish if the same results will hold.

\section{Acknowledgement}

Our ultimate appreciation goes to the Almighty, God for making our project work a successful one.

The next appreciation goes to our project supervisor $\mathrm{Mr}$. Ofosu Addo Henry for his dedication and guidance and most of all his support in ensuring that this work was completed and at the acceptable standard.

The Nurses and other health Professionals of the Dormaa 
Presby Hospital, the Researchers thank you for your dedication and kind assistance in administering the questionnaire as well as for Continuing to discharge your duties.

Also, to the clients of Dormaa Presby Hospital Diabetic Clinic, for agreeing to participate in this study as without them, this study would not have been conceptualized.

We thank our families and friends for the spiritual help, support and putting up with long hours we spent behind the computer producing this work.

\section{References}

[1] Adil, M. M., Alam, A. Y., \& Jaffery, T. (2005 ). Knowledge of Type 2 Diabetic Patients about their illness. JPMA, pp. 57-78.

[2] Alan, M. (2011), The role of health beliefs in the regimen adherence and metabolic control of adolescents and adults with diabetes mellitus. J Consult Clin Psycho, pp. 139-144.

[3] Anderson, R., Funnell, M., Burkhart, N., Gillard, M., \& Nwankwo, R. 101 Tips for Behavior Change in Diabetes Education. Alexandria, VA: American Diabetes Association, 2008.

[4] Arauz AG, S. G. (2012). Community diabetes educational intervention at the primary care level. Rev Panam Salud Publica, pp. 145-53.

[5] Arifulla, M., John, L. J., Sreedharan, j., Muttappallymyalil, J., \& Basha, a. S. (2014). Patients' Adherence to Anti-Diabetic Medications in a Hospital at Ajman, UAE. Malays J Med Sci, volume21 Num.1, pp. 44-49.

[6] CDC, (2011). Distribution of age at diagnosis of diabetes among adults, diabetes public health resource. Atlanta GA.

[7] Claxton AJ, C. J. (2009). A systematic review of the associations between dose regimens and medication compliance. Clin Ther volume23 Num.(8), pp. 1296-1310.

[8] Claxton AJ. (2009). A systematic review of the association between dose. BMJ VOL66, pp. 45-78.

[9] Delamater, A. M. (2006). Improving Patient Adherence. Clinical Diabetes vol. 24, pp. 71-77.

[10] Glasgow RE, T. D. (2009). Social environment and regimen adherence among type II diabetic patients. Diabetes Care volume 11 , pp. 377-386.

[11] Harris MI, C. C. (1993). Self-monitoring of blood glucose by adults with diabetes in the United States population. Diabetes Care volume 16, pp. 1116-1123.

[12] Haynes RB, T. D. (2012). Compliance in health care. Baltimore: Johns Hopkins University Press.
[13] Horne R, W. J. (2007). Patients' beliefs about prescribed medicines and their role in adherence to treatment in chronic physical illness. J Psychosom Res, pp. 555-567.

[14] Joan N Kalyango, E. O. ( 2008). Non-adherence to diabetes treatment at Mulago Hospital in Uganda: prevalence and associated factors. Afr Health Sci. volume 8 Num.2, pp. 67-73.

[15] Larme A, P. J. (2011). Attitudes of primary care providers toward diabetes: barriers to guideline implementation. Diabetes Care volume21, pp. 1391-1396.

[16] M, H. (2009). Non-communicable diseases, Community Medicine and Public Health, oth ed. Karachi: Time Publisher.

[17] McDonald HP, G. A. (2011). Intervention to enhance patient adherence to medication. JAMA, pp. 2868-2879.

[18] Meichenbaum D, T. D. (2013). Facilitating Treatment Adherence: A Practitioner's Guide-book. New York: Plenum Press.

[19] Odegard PS, C. K., \& D, F. (2007). Medication taking and diabetes: a systematic review of the literature. Diabetes Educ VOL 33 Num.6, pp.1014-1029.

[20] Ohene Buabeng K, M. L.-R. (2008). Unaffordable drug prices: the major cause of noncompliance with diabetic medication in Ghana. J Pharm Sci vol.7 Numr3, pp. 350-352.

[21] Osterberg L, B. T. (2007). Adherence to medication. $N$ Engl J Med, pp. 487-497.

[22] Paes AHP, B. A.-A. (2007). Impact of dosage frequency on patient compliance. Diabetes Care 20, pp. 1512-1517.

[23] Peyrot M, R. R. (2010). Psychosocial problems and barriers to improved diabetes management: results of the Cross-National Diabetes Attitudes, Wishes and Needs (DAWN) Study. Diabet Med, pp. 1379-1385.

[24] Rollnick S, M. P. (2013). Health Behavior Change: A Guide for Practitioners. Edinburgh, U.K: Churchill Livingstone.

[25] Simpson SH, E. D. (2008). A meta-analysis of the association between adherence to drug therapy and mortality. BMJ vol 15 , pp. 333-7557.

[26] US Dept of Health and Human Services. (2008, November 15). Retrieved May 10, 2014, from US Dept of Health and Human Services Web site: http://www.health.gov/communication/literacy/issuebrief/

[27] Winkler A, T. A. (2010). Monitoring adherence to prescribed medication in type 2 diabetic patients treated with sulfonylureas. Swiss Med Wkly vol 44, pp. 34-99.

[28] World Health Organization. (2010). besity: preventing and managing the global epidemic. Technical Report Series No. 894. Geneva: WHO. 